\title{
Cutoff conditions for transverse circularly polarized electromagnetic waves in a finite temperature electro-magneto-ionic medium
}

\author{
H. C. HsIeH \\ Electron Physics Laboratory, Department of Electrical Engineering, \\ The University of Michigan, Ann Arbor, Michigan, U.S.A.
}

(Received 7 February 1967; in revised form 5 April 1967)

\begin{abstract}
The dispersion relation for wave propagation in a homogeneous, electrically neutral electron gas subject to crossed static electric and magnetic fields is derived using the coupled Maxwell-Boltzmann-Vlasov equations. The cutoff condition for transverse circularly polarized electromagnetic waves is obtained from the derived dispersion relation. The variation of the cutoff frequency $\omega_{0}$ with the static electric field $E_{0}$, magnetostatic field $B_{0}$, the electron number density $N$ and the electron gas temperature $T$ is discussed. For example it is shown that for a givon value of $B_{0}$ and $N$, eithor a decrease of the electron gas temperature $T$ or an increase of static electric field $E_{0}$ will cause the cutoff frequency of the left-hand circularly polarized wave to increase while the cutoff frequency of the right-hand circularly polarized wave decreases. A possible application of the theory to the study of electromagnetic wave propagation in the region of ionospheric plasma, where both static electric and static magnetic fields are present, is indicated.
\end{abstract}

\section{INTRODUCTION}

IT Is well known that the propagation of electromagnetic waves in a magnetoionic medium can be characterized by the Appleton equation. A detailed discussion on this subject has been given by RATCLIFFE (1959). The magnetoionic medium is defined as one in which free electrons and heavy ions are situated in a uniform magnctic field and are distributed with statistical uniformity, so that there is no resultant space charge.

The cutoff conditions for the circularly polarized electromagnetic wave in a cold plasma have been discussed in detail by HEALD and WHARTON (1965). Recently, in the course of investigating the effect of a transverse static electric field on the propagation of the circularly polarized electromagnetic wave in a finite temperature plasma, this author (HsIEH, 1966) has shown that the presence of a static electric field may cause the cutoff frequency of the electromagnetic wave to shift. The purpose of this paper, therefore, is to discuss the cutoff conditons for the circularly polarized waves in a homogeneous, electrically neutral electron gas, subjected to crossed electrostatic and magnetostatic fields. This system is referred to as the electro-magneto-ionic medium. The thermal motion of an electron is considered although ion motion and collision effects are assumed to be negligible. The present discussion is based on a small-amplitude, one-dimensional analysis in which all time-varying quantities are assumed to have harmonic dependence of the form: $\exp [j(\omega t-k z)]$, where $\omega$ and $k$ are the wave angular frequency and propagation constant respectively. $z$ and $t$ denote, respectively, the spatial and time variables. 


\section{Dispersion Relations}

Consider all quantities of interest to be composed of two parts; the time-independent part denoted by the subscript " 0 " and the time-dependent part denoted by the subscript "l", e.g. the magnetic flux density $\mathbf{B}$ and electric field intensity $\mathbf{E}$ are written as

$$
\mathbf{B}=\mathbf{B}_{0}(z)+\mathbf{B}_{1}(z, t) \text { and } \mathbf{E}=\mathbf{E}_{0}(z)+\mathbf{E}_{\mathbf{1}}(z, t)
$$

and the electron distribution function $f$ is written as

$$
f=f_{0}(z, \mathbf{v})+f_{1}(z, \mathbf{v}, t) .
$$

Suppose that the applied static electric field $\mathbf{E}_{a}$ and magnetostatic field $\mathbf{B}_{\mathbf{0}}$ are directed along the positive $y$ - and positive $z$-directions respectively.

The dynamic electromagnetic fields in the electron gas are governed by Maxwell's field equation, which is expressed in the following manner:

$$
\begin{aligned}
& \mathbf{E}_{ \pm}=\frac{-j\left(\frac{\omega e}{\varepsilon_{0}}\right)}{\left(\omega^{2}-c^{2} k^{2}\right)} \int v_{r} \mathrm{e}^{ \pm j \rho} f_{1} d^{3} v, \\
& E_{1 z}=\frac{-j e}{\omega \varepsilon_{0}} \int v_{z} f_{1} d^{3} v
\end{aligned}
$$

where $c=1 / \sqrt{ }\left(\mu_{0} \varepsilon_{0}\right)$ is the speed of light in free space, and $\mu_{0}$ and $\varepsilon_{0}$ denote the permeability and dielectric constant of vacuum; $d^{3} v=\left(v_{r} d v_{r} d \varphi d v_{z}\right)$ is a volume element in velocity space. On the other hand, the electron distribution is described by the Boltzmann-Vlasov equation, which is written as

$$
\begin{aligned}
& \left(j\left(\omega-k v_{z}\right)+\omega_{z} \frac{\partial}{\partial \varphi}\right) f_{1}-a_{--}\left(\frac{\partial f_{1}}{\partial v_{r}}+\frac{j}{v_{r}} \frac{\partial f_{1}}{\partial \varphi}\right) \mathrm{e}^{j \varphi}-a_{+}\left(\frac{\partial f_{1}}{\partial v_{z}}-\frac{j}{v_{r}} \frac{\partial f_{1}}{\partial \varphi}\right) \mathrm{e}^{-i \varphi} \\
& =\frac{e}{m} M_{-}\left(f_{0}\right) E_{-} \mathrm{e}^{j \varphi}{ }_{m}^{e} M_{+}\left(f_{0}\right) E_{+} \mathrm{e}^{-j \varphi}{ }_{m}^{e} E_{1 z} \frac{\partial f_{0}}{\partial v_{z}},
\end{aligned}
$$

where

$$
\begin{aligned}
E_{\mp} & -\frac{1}{2}\left(E_{1 x} \mp j E_{1 y}\right), B_{\mp}-\frac{1}{2}\left(B_{1 x} \mp j B_{1 y}\right), \\
a_{\mp} & =\mp j \frac{e E_{a}}{2 m}, \quad \omega_{z} \equiv\left(\frac{\mathrm{e} B_{0}}{m}\right), \\
M_{\mp}\left(f_{0}\right) & =\left[\left(1-\frac{k v_{z}}{\omega}\right)\left(\frac{\partial f_{0}}{\partial v_{r}} \mp \frac{j}{v_{r}} \frac{\partial f_{0}}{\partial \varphi}\right)+\frac{k v_{r}}{\omega} \frac{\partial f_{0}}{\partial v_{z}}\right], \\
v_{x} & =v_{r} \cos \varphi, \quad v_{y}=v_{r} \sin \varphi .
\end{aligned}
$$

Here $E_{-}$and $E_{+}$denote the left- and right-hand eircularly polarized components of electric field respectively. $E_{1 z}$ is the longitudinal component of electric field. $\omega_{z}$ is the electron cyclotron frequency.

Suppose that the time-varying distribution function $f_{1}$ is written in the form.

$$
f_{1}\left(z, t, v_{r}, v_{z}, \varphi\right)=f_{-}\left(z, t, v_{r}, v_{z}\right) \mathrm{e}^{j \varphi}+f_{+}\left(z, t, v_{r}, v_{z}\right) \mathrm{e}^{-j \varphi}+g\left(z, t, v_{r}, v_{z}\right),
$$


in which the first, second and third terms of the right-hand side can be regarded as the left-hand circularly polarized, the right-hand circularly polarized wave, and the longitudinal components of the distribution function respectively. Then in view of the fact that equation (2) must be valid for an arbitrary value of $\varphi$, the substitution of equation (4) into equation (2) yields a system of equations, expressing the functions $f_{-}, f_{+}$and $g$ in terms of the electric field components $E_{-}, E_{+}$and $E_{1 z}$ as follows:

$$
\begin{aligned}
f_{-} & =k_{11} E_{-}+k_{12} E_{+}+k_{13} E_{1 z}, \\
f_{+} & =k_{21} E_{-}+k_{22} E_{+}+k_{23} E_{1 z}, \\
g & =k_{31} E_{-}+k_{32} E_{+}+k_{33} E_{1 z},
\end{aligned}
$$

where

$$
\begin{aligned}
& k_{11}=\frac{-j \frac{\mathrm{e}}{m} M_{-}\left(f_{0}\right)}{\left(b+\omega_{z}\right)}, \quad k_{12}=0, \quad k_{13}=\frac{-\frac{\mathrm{e}}{m} a_{-} \frac{\partial}{\partial v_{r}}\left(\frac{\partial f_{0}}{\partial v_{z}}\right)}{b\left(b+\omega_{z}\right)} \\
& k_{21}=0, \quad k_{22}=\frac{-j \frac{\mathrm{e}}{m} M_{+}\left(f_{0}\right)}{\left(b-\omega_{z}\right)}, \quad k_{23}=\frac{-\frac{\mathrm{e}}{m} a_{+} \frac{\partial}{\partial v_{r}}\left(\frac{\partial f_{0}}{\partial v_{z}}\right)}{b\left(b-\omega_{z}\right)} \\
& k_{31}=\frac{-2 \frac{\mathrm{e}}{m} \frac{a_{+}}{v_{r}} M_{-}\left(f_{0}\right)}{b\left(b+\omega_{z}\right)}, \quad k_{32}=\frac{-2 \frac{\mathrm{e}}{m} \frac{a_{-}}{v_{r}} M_{+}\left(f_{0}\right)}{b\left(b-\omega_{z}\right)}, \\
& k_{33}=\frac{-j \frac{\mathrm{e}}{m} \frac{\partial f_{0}}{\partial v_{z}}}{b}+j \frac{4 a_{-} a_{+}}{v_{r}} \frac{\mathrm{e}}{b\left(b^{2}-\frac{\partial}{\partial v_{r}} \frac{\partial f_{0}}{\partial v_{z}}\right.}, \quad b \equiv\left(\omega-k v_{z}\right) .
\end{aligned}
$$

Upon combining equations ( 1 ) and (5), the following set of algebraic equations governing the electric field components is obtained:

where

$$
\begin{aligned}
& E_{-}=R_{11} E_{-}+R_{12} E_{+}+R_{13} E_{1 z}, \\
& E_{+}=R_{21} E_{-}+R_{22} E_{+}+R_{23} E_{1 z}, \\
& E_{1 z}=R_{31} E_{-}+R_{32} E_{+}+R_{33} E_{1 z},
\end{aligned}
$$

$$
\begin{aligned}
& R_{1 l}=\frac{-j\left(\frac{\omega \mathrm{e}}{\varepsilon_{0}}\right)}{2\left(\omega^{2}-c^{2} k^{2}\right)} \int_{-\infty}^{\infty} \int_{0}^{\infty} v_{r}^{2} \int_{0}^{2 \pi}\left(k_{1 l}+k_{2 l} \mathrm{e}^{-j 2 \varphi}+k_{3 l} \mathrm{e}^{-j \varphi}\right) d \varphi d v_{r} d v_{z}, \\
& R_{2 p}=\frac{-j\left(\frac{\omega \mathrm{e}}{\varepsilon_{0}}\right)}{2\left(\omega^{2}-c^{2} k^{2}\right)} \int_{-\infty}^{\infty} \int_{0}^{\infty} v_{r}^{2} \int_{0}^{2 \pi}\left(k_{1 p} \mathrm{e}^{j 2 \varphi}+k_{2 p}+k_{3 p} \mathrm{e}^{j \varphi}\right) d \varphi d v_{r} d v_{z}, \\
& R_{3 q}=\frac{-j e}{\omega \varepsilon_{0}} \int_{-\infty}^{\infty} v_{z} \int_{0}^{\infty} v_{r} \int_{0}^{2 \pi}\left(k_{1 q} \mathrm{e}^{j \varphi}+k_{2 q} \mathrm{e}^{-j \varphi}+k_{3 q}\right) d \varphi d v_{r} d v_{z},
\end{aligned}
$$

in which $l, p$, and $q$ take the values of 1,2 , and 3. The coefficients $k_{p q}$ are given in equations (6). 
The dispersion relation for the system is readily given from equation (7) and is expressed as

$$
D(\omega, k)=\left|\begin{array}{lll}
\left(R_{11}-1\right) & R_{12} & R_{13} \\
R_{21} & \left(R_{22}-1\right) & R_{23} \\
R_{31} & R_{32} & \left(R_{33}-1\right)
\end{array}\right|=0 .
$$

It should be noted that equations (7) indicate the possibility of coupling between the characteristic modes. Once the time-independent distribution function $f_{0}$ is known, then the coefficients $k_{p q}$ are determined so that $R_{p q}$ can be evaluated. $f_{0}$, which must satisfy the time-independent part of the Boltzmann-Vlasov equation for the case of a homogeneous, electrically neutral electron gas subjected to crossed static electric and magnetic fields, can be written in a Maxwellian form as follows:

$$
\left.f_{0}=n_{0} \exp \left\{-\alpha \mid\left(v_{x}-u\right)^{2}+v_{y}{ }^{2}+v_{z}{ }^{2}\right]\right\},
$$

where $\alpha \equiv(m / 2 K T)$ and $u \equiv\left(E_{0} / B_{0}\right) . K$ is the Boltzmann constant, $T$ and $u$ denote the temperature and drift velocity of an electron respectively, and $n_{0}$ is the normalization constant.

Based on equation (9) the elements of the determinant in dispersion relation (8) can be given as follows:

$$
\begin{aligned}
& R_{11}=\underset{(1-\eta)}{X}\left[\begin{array}{c}
1 \\
1+Y+{ }_{2}^{\gamma}
\end{array}\left(\begin{array}{lcc}
\delta & 1 & 1 \\
& (1+Y)^{3}
\end{array}\right)\right], \\
& R_{12}=\frac{\delta X}{(1-\eta)}\left[\left(1+\frac{\partial}{2} \frac{Y}{(1-Y)}\right)+\frac{\gamma}{2}\left(1+\frac{\delta}{2(1-Y)^{3}}\right)\right], \\
& R_{13}=\frac{\sqrt{ }(\delta \gamma) X}{2(1-\eta)}\left[\left(2-\frac{\delta}{2}\right)+\frac{\left(\frac{\delta}{2}\right)-1}{(1+Y)^{2}}\right] \\
& R_{21}=\frac{\delta X}{(1-\eta)}\left[\left(1-\frac{\delta}{21} \frac{Y}{1+Y}\right)+\frac{\gamma}{2}\left(1-\frac{\delta}{2} \frac{Y}{2(1+Y)^{3}}\right)\right], \\
& R_{22}=\frac{X}{(1-\eta)}\left[\frac{1}{1-\mathrm{Y}}+\frac{\gamma}{2}\left(\delta+\frac{1}{(1-Y)^{3}}\right)\right] \text {, } \\
& R_{23}=\frac{\sqrt{ }(\delta \gamma) X}{2(1-\eta)}\left[\left(2-\frac{\delta}{2}\right)+\frac{(\delta / 2-1)}{(1-\mathrm{Y})^{2}}\right] \text {, } \\
& R_{31}=\sqrt{ }(\delta \gamma) X\left(1-\frac{\delta}{2} \frac{(1-\delta) Y}{(1+Y)^{2}}\right) \text {, } \\
& R_{32}=\sqrt{ }(\delta \gamma) X\left(1+\frac{\partial(1-\delta) Y}{2(1-Y)^{2}}\right), \\
& R_{33}=X\left[1+\frac{\delta\left(1-\frac{3}{2} \delta\right) Y^{2}}{\left(1-Y^{2}\right)}\right] \text {, }
\end{aligned}
$$


where

$$
\begin{gathered}
X \equiv\left(\frac{\omega_{p}}{\omega}\right)^{2}, \quad Y \equiv\left(\frac{\omega_{z}}{\omega}\right), \quad \eta \equiv\left(\frac{c^{2} k^{2}}{\omega^{2}}\right), \\
\gamma \equiv \frac{1}{\alpha v_{0}^{2}}, \quad \delta \equiv \alpha u^{2}, \quad v_{0} \equiv\left(\frac{\omega}{k}\right), \quad \alpha \equiv\left(\frac{m}{2 K T}\right),
\end{gathered}
$$

and provided that

$$
\delta^{2} \ll 1, \quad \gamma^{2} \ll 1 \text { and }\left|\sqrt{ }\left(\alpha v_{0}\right)(1 \pm Y)\right|^{4} \gg 1
$$

The assumption that conditions (11) are satisfied is equivalent to assuming that the drift velocity $u$ is much smaller than the thermal velocity, while the thermal velocity is much smaller than the phase velocity of the transverse electromagnetic wave.

It is of interest to note that as $\delta \rightarrow 0, R_{p q} \rightarrow 0$, when $p \neq q$, so that the offdiagonal terms of the determinant in equation (8) vanish, which suggests that the coupling between the modes disappears. In this case equation (8) becomes

$$
\left(R_{11}-1\right)\left(R_{22}-1\right)\left(R_{33}-1\right)=0,
$$

which in turn yields three dispersion equations for the uncoupled characteristic modes, as shown in equation (13):

$$
\begin{gathered}
1=\frac{X}{(1-\eta)}\left(\frac{1}{1+Y}+\frac{\gamma}{2} \frac{1}{(1+Y)^{3}}\right) \\
1=\frac{X}{(1-\eta)}\left(\frac{1}{1-Y}+\frac{\gamma}{2} \frac{1}{(1-Y)^{3}}\right) \\
1=X .
\end{gathered}
$$

Equations (13) are, rspectively, the dispersion equations for the left-hand circularly polarized wave, the right-hand circularly polarized wave, and the longitudinal plasma oscillation. It should be noted that as $\gamma \rightarrow 0$, which is the case when $T \rightarrow 0$, equations (13a) and (13b) are reduced to the familar expression in the cold-plasma magnetoionic theory.

\section{The Cutofy Condrtons for the Transuerse Electro- MAGNETIO WaVES}

The cutoff of electromagnetic wave propagation occurs when its propagation constant $\mathrm{k}$ becomes zero, in which case $\eta=0$ and $\gamma=0$ so that, from equation (10), $R_{13}=R_{23}=R_{31}=R_{32}=0$. Equation (8) becomes

$$
\left[\left(r_{11}-1\right)\left(r_{22}-1\right)-r_{12} r_{21}\right]\left(R_{33}-1\right)=0 \text {, }
$$

where $r_{p q}$ is the value of $R_{p q}$ for $\eta=0$ and $\gamma=0$. Therefore the cutoff conditions for the transverse electromagnetic wave are given by

$$
\left(r_{11}-1\right)\left(r_{22}-1\right)-r_{12} r_{21}=0
$$

which can be conveniently written as

$$
\frac{\delta^{2} y_{0}{ }^{2}(1-\delta)}{x^{4}}=y_{0}^{2}-\left(x-\frac{1}{x}\right)^{2}
$$


where $y_{0} \equiv\left(\omega_{z} / \omega_{p}\right)$ is the ratio of the electron cyclotron frequency $\omega_{z}$ to the electron plasma frequency $\omega_{p}$ and $x \equiv\left(\omega_{0} / \omega_{p}\right)$ with $\omega_{0}$ being the cutoff frequency of the transverse electromagnetic wave. Once the values of $y_{0}$ and $\delta$ are sperified, equation (16) can be solved for $x$ so that $\left(\omega_{0} / \omega_{p}\right)$ is determined. However, the variation of $\omega_{0}$ with respect to $\delta$ can easily be observed with the aid of a graphical method illustrated below:

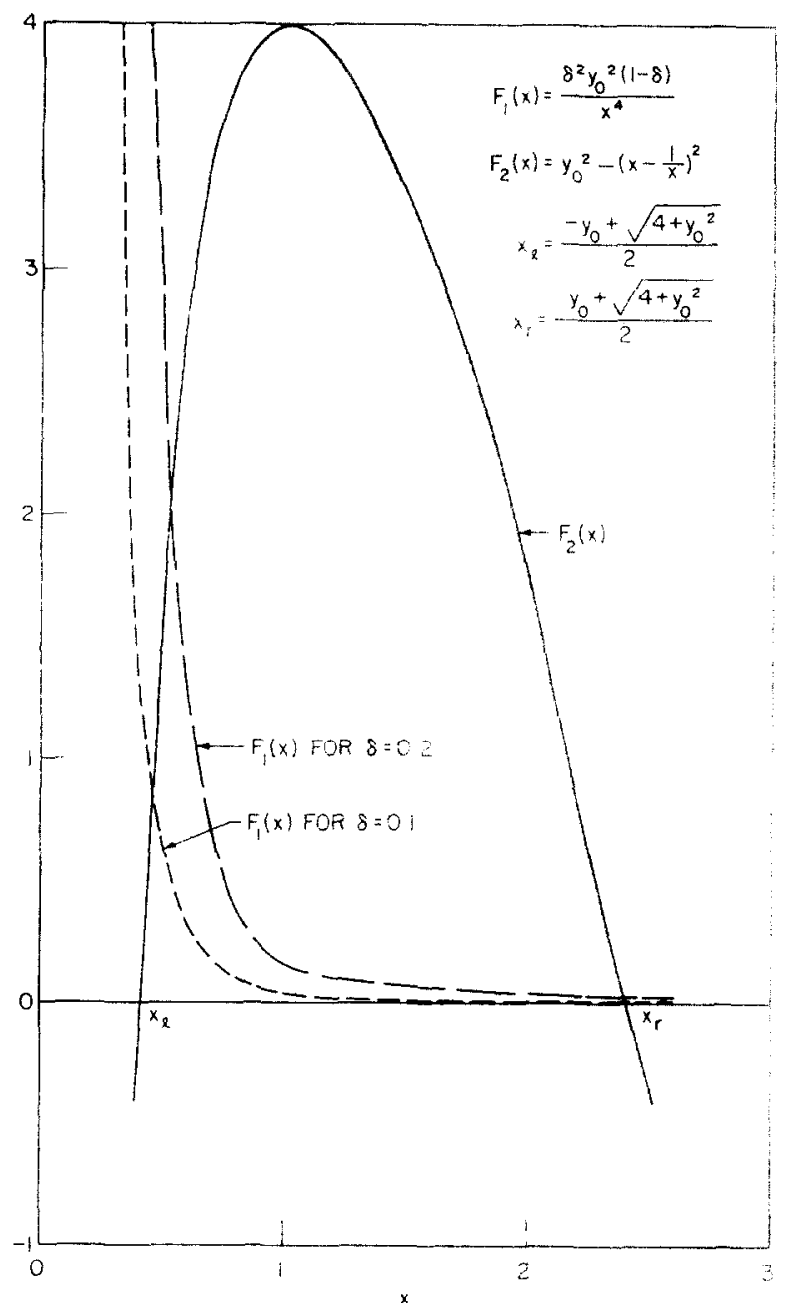

Fig. 1. Illustration of graphical solution of equation (16).

Let $F_{1}(x)$ be the left-hand side and $F_{2}(x)$ be the right-hand side of equation (16). If $F_{1}$ vs. $x$ and $F_{2}$ vs. $x$ are plotted in the same plane, as illustrated in Fig. 1, then the intersections of the two plots provides the real roots of equation (16). Once $y_{0}$ is given, the curve of $F_{2}(x)$ is determined, and if $\delta$ is also specified, then $F_{1}(x)$ is completely determined. Thus the intersection point of the two plots is readily determined. It should be noted that when $\delta=0$ the $F_{1}$ curve coincides with the $x$-axis. and if its 
intersections with the $F_{2}$ curve are denoted by $x_{l}$ and $x_{r}$, then they are given by

$$
x_{l}=\frac{-y_{0}+\sqrt{ }\left(y_{0}^{2}+4\right)}{2} \text { and } x_{r}=\frac{y_{0}+\sqrt{ }\left(y_{0}^{2}+4\right)}{2} .
$$

$x_{l}$ determines $\omega_{0 l}$, the cutoff frequency of the left-hand circularly polarized wave, and

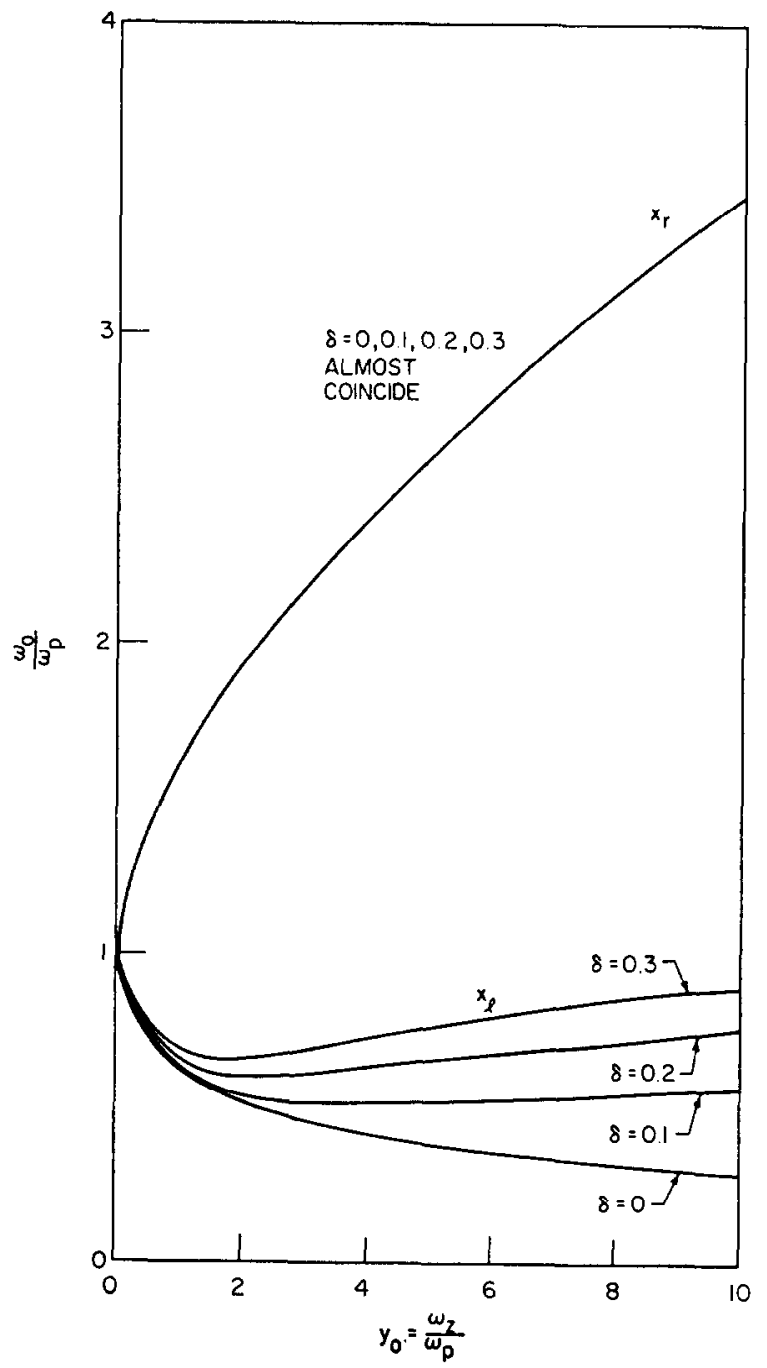

Fig. 2. The plot of $\left(\omega_{0} / \omega_{p}\right)$ vs. $\left(\omega_{z} / \omega_{p}\right)$ with $\delta$ as parameter.

$x_{r}$ determines $\omega_{0 r}$, the cutoff frequency of the right-hand circularly polarized wave. It is easily seen from Fig. I that an increase in the parameter $\delta$ leads to an increase in $\omega_{02}$, but to a slight decrease in $\omega_{0 r}$. Numerical illustration of equation (16) is given in Figs. 2-4. The normalized cutoff frequency $x=\left(\omega_{0} / \omega_{p}\right)$ as a function of the normalized cyclotron frequency $y_{0}=\left(\omega_{z} / \omega_{y}\right)$ for various values of the parameter $\delta=$ $(m / 2 K T)\left(E_{0}{ }^{2} / B_{0}{ }^{2}\right)$ is shown in Fig. 2. The plot of $x$ vs. $\delta$ for various values of $y_{0}$ is 

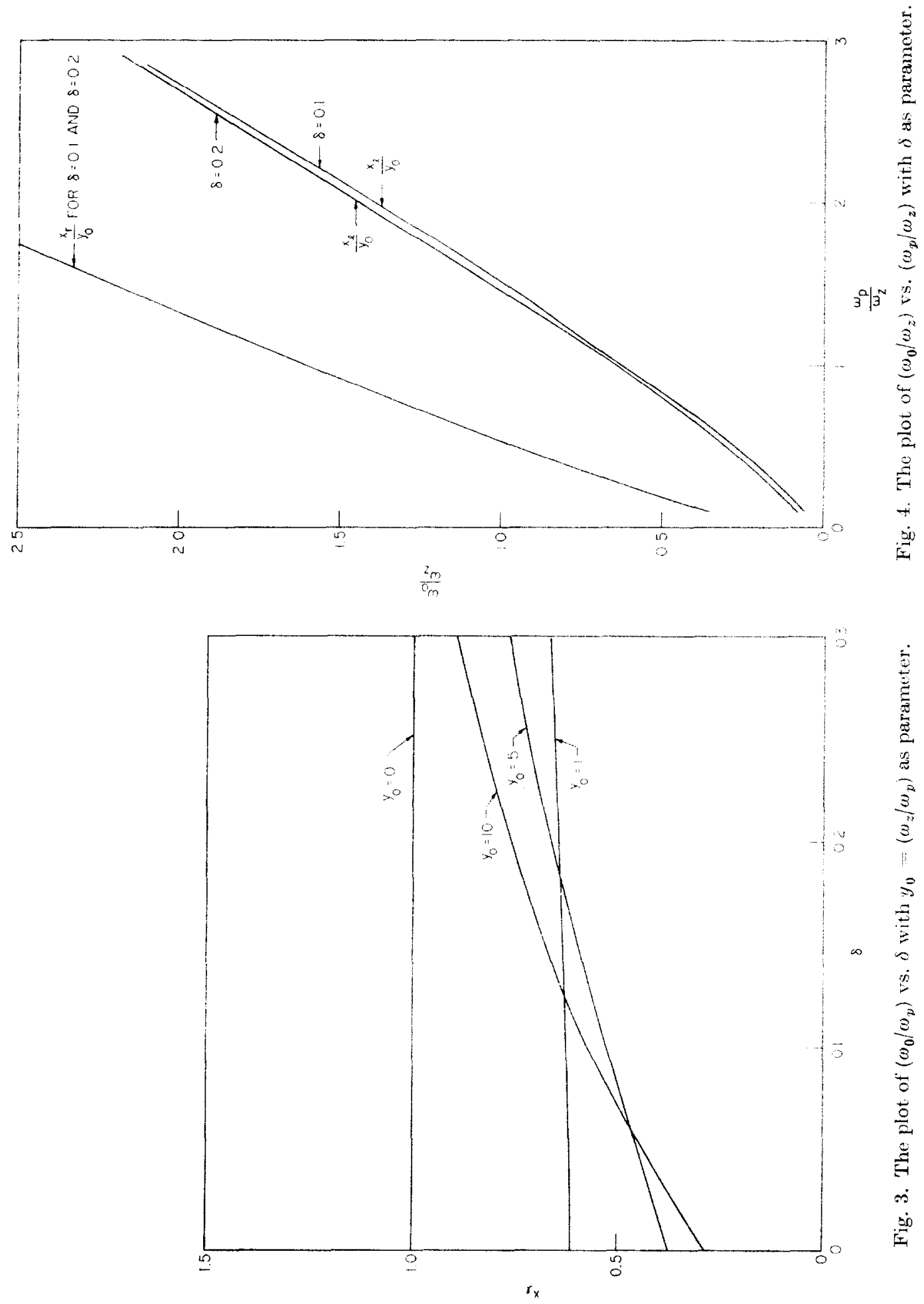

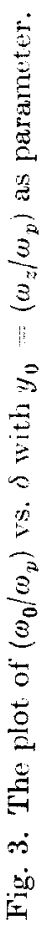


shown in Fig. 3, and the plot of $\left(\omega_{0} / \omega_{z}\right)$ vs. $\left(\omega_{p} / \omega_{z}\right)$ for various values of $\delta$ is shown in Fig. 4.

It is of interest to observe that in Fig. $2 x_{r}$ increases with $y_{0}$ while $x_{l}$ decreases with $y_{0}$ in the range of small $y_{0}$, for the case $\delta>0 \cdot 1$, and increases with $y_{0}$ in the range of large $y_{0}$. A change in the value of $\delta$ appears to affect the value of $x_{l}$ significantly, but has only a slight effect on $x_{r}$. The variation of $x_{l}$ with $\delta$ for a given value of $y_{0}$ is shown in Fig. 3. $x_{l}$ appears to increase with $\delta$ faster for the larger value of $y_{0}$ than for the small value of $y_{0}$. It is observed in Fig. 4 that for a given $B_{0}$ and $\delta$, the cutoff frequency of transverse electromagnetic waves increases with the electron density monotonically. In view of the fact that $\delta$ is proportional to the square of the static electric field $E_{0}$ and inversely proportional to the temperature $T$, the above observations suggest that for a given $B_{0}$ either a decrease in $T$ or an increase in $E_{0}$ will cause $\omega_{0 l}$ to increase while $\omega_{0 r}$ decreases slightly in the range of the value of $y_{0}$ chosen.

\section{Concluding Remarks}

It should be noted that the cutoff frequency $\omega_{0}$ in the cutoff condition, equation (16), is expressed in terms of the electron cyclotron frequency $\omega_{z}$, the electron plasma frequency $\omega_{p}$, and $\delta$ which is the square of the ratio of the electron drift velocity $\left(E_{0} / B_{0}\right)$ to the thermal velocity $\sqrt{ }(2 K T / m)$. Since $\omega_{z}$ is proportional to the static magnetic field $B_{0}$ and $\omega_{p}$ is proportional to $\sqrt{ } N$, with $N$ being the number density, equation (16) gives the relation between $\omega_{0}, E_{0}, B_{0}, N$ and $T$. Suppose that $E_{0}, B_{0}$, and $N$ are known in the region of an electron gas under consideration; then by observing the cutoff frequency of a circularly polarized electromagnetic wave, the temperature $T$ of the electron gas can be, in principle, determined with the aid of equation (16). This suggests a possible diagnostic technique for the measurement of electron gas temperature.

On the other hand, most analyses of electromagnetic wave propagation in the ionospheric plasma in the past appear to have been concerned primarily with the effect of the Earth's magnetic field, but little or no attention has been given to the effect of static electric field which may be present in the ionosphere. However, the existence of electrostatic fields in the ionosphere and magnetosphere has been postulated by various workers in the studies of various ionospheric phenomena, for example, in the formation of $F$-region irregularities (e.g. DAGG, 1957; FARLEY, 1959, 1960 ; SPREITER and Brigas, 1961).

The method of analysis developed in this paper can be profitably applied to the investigation of transverse circularly polarized electromagnetic wave propagation along the geomagnetic field line in the ionospheric plasma in which the presence of a transverse static electric field may be important. For example, a whistler propagating along the geomagnetic field line between two hemispheres might encounter a region of the ionosphere or magnetosphere in which a static electric field is perpendicular to the geomagnetic field. Then a natural question arises as to what is the effect of this static electric field on the propagation characteristics of electromagnetic waves, if there is any? To answer this type of question e.g. dispersion equation (8) together with equations (10) can be used.

Furthermore it should be pointed out that it is not difficult to extend the present method of analysis to include the effect of ion motion, as well as collisions. In this 
case the element $R_{p q}$ in equations 10 and the cutoff condition will be modified. The application of the theory developed in this paper to the ionospheric plasma is to be considered in detail in a future paper.

Acknowledgements - The author is very grateful to Professor J. L. Rowe for his contributions and for reading the manuscript. The work was supported by the National Aeronautics and Space Administration under Grant No. NsG 696.

DAGG M.

Farley D. T.

Farley D. T.

Heard M. A. and Wharton C. B.

Hsieh H. C.

RATCLIFFE J. A.

Spreiter J. R. and Briggs B. R.

\section{REFERENCES}

1957 厅. Atmosph. Terr. Phys. 11, 139.

$1959 \quad J$. Geophys. Res. 64, 1225.

1960 I. Geophys. Res. 65, 869.

1965 Plasma Diagnostics with Microwaves, Chap. 1, John Wiley, New York.

1966 A paper presented at 8th A. Mtg. Am. Physic. Soc., Dir. Plasma Phys., Boston, Mass., November 2-5.

1959 The Magneto-ionic Theory and its Applications to the Ionosphere, University Press, Cambridge, Mass.

1961 J. Geophys. Res. 66, 1731. 